\title{
EXACT BOUNDARY CONTROLLABILITY FOR THE KORTEWEG-DE VRIES EQUATION ON A BOUNDED DOMAIN
}

\author{
LIONEL ROSIER
}

\begin{abstract}
The exact boundary controllability of linear and nonlinear Korteweg-de Vries equation on bounded domains with various boundary conditions is studied. When boundary conditions bear on spatial derivatives up to order 2, the exact controllability result by Russell-Zhang is directly proved by means of Hilbert Uniqueness Method. When only the first spatial derivative at the right endpoint is assumed to be controlled, a quite different analysis shows that exact controllability holds too. From this last result we derive the exact boundary controllability for nonlinear KdV equation on bounded domains, for sufficiently small initial and final states.
\end{abstract}

\section{INTRODUCTION AND MAIN RESULTS}

The Korteweg-de Vries (KdV) equation

$$
y_{t}+y y_{x}+y_{x x x}=0,
$$

is a well known instance of a nonlinear dispersive partial differential equation, which may serve as a model for (among other things) propagation of small amplitude long water waves in a uniform channel.

Recently stabilizability and controllability results have been obtained in [8] for the forced Korteweg - de Vries equation with periodic boundary conditions

$$
\begin{aligned}
y_{t}+y y_{x}+y_{x x x} & =f(t, x), \quad 0<x<1,0<t<T \\
\frac{\partial^{k} y}{\partial x^{k}}(t, 0) & =\frac{\partial^{k} y}{\partial x^{k}}(t, 1), \quad 0 \leq k \leq 2
\end{aligned}
$$

the control action $f(t, x)$ being localized:

$$
\operatorname{supp} f(t, .) \subset[a, b] \subset[0,1] .
$$

On the other hand, there are to date few results concerning the boundary controllability of KdV equation. In [16] boundary controllability of linear

Laboratoire d'analyse numérique, Université d'Orsay, bât. 425, 91405 Orsay, France. E-Mail: Lionel.ROSIER@math.u-psud.fr.

Received by the journal April 16, 1996. Revised November 22, 1996. Accepted for publication December 27, 1996.

This research was funded by NSF grant DMS 9530973.

(C) Société de Mathématiques Appliquées et Industrielles. Typeset by LATEX. 
KdV equation

$$
\begin{aligned}
y_{t}+y_{x x x} & =0, \quad 0<x<2 \pi, \quad 0<t<T \\
\frac{\partial^{k} y}{\partial x^{k}}(t, 0) & =\frac{\partial^{k} y}{\partial x^{k}}(t, 2 \pi), \quad k \in\{0,2\} \\
\frac{\partial y}{\partial x}(t, 2 \pi) & -\frac{\partial y}{\partial x}(t, 0)=h(t), \quad 0<t<T,
\end{aligned}
$$

is obtained as a consequence of the uniform stabilizability of this timereversible (linear) system in both t-directions, whose proof is rather long and technical. Moreover only initial and terminal states $y_{0}$ and $y_{T}$ for which

$$
\int_{0}^{2 \pi}\left(y_{T}(x)-y_{0}(x)\right) d x=0
$$

are concerned and the control $h(t)$ is not explicitly given. The first goal of this paper is to directly study the (exact) boundary controllability problem for linear KdV equation by means of the Hilbert Uniqueness Method (H.U.M). This approach has been successfully applied for studying controllability of wave and plate equations, and more recently of Schrödinger equation (see for instance [1], [5], [7], [11], [12], [13], [14]). The first result of this paper concerning boundary controllability of linear KdV equation is as follows:

Theorem 1.1. Let $H_{p}^{2}=\left\{w \in H^{2}(0,2 \pi): w(0)=w(2 \pi) ; w^{\prime}(0)=w^{\prime}(2 \pi)\right\}$ and $T>0$. Then for any $y_{0}, y_{T} \in\left(H_{p}^{2}\right)^{\prime}$ (the dual space of $\left.H_{p}^{2}\right)$, there exist $h_{0}, h_{1}, h_{2} \in L^{2}(0, T)$ such that the solution $y \in C\left([0, T],\left(H_{p}^{2}\right)^{\prime}\right)$ of the boundary initial-value KdV equation:

$$
\begin{aligned}
y_{t}+y_{x x x} & =0, \quad(x, t) \in(0,2 \pi) \times(0, T) \\
y(t, 2 \pi)-y(t, 0) & =h_{0}(t), \\
y_{x}(t, 2 \pi)-y_{x}(t, 0) & =h_{1}(t), \\
y_{x x}(t, 2 \pi)-y_{x x}(t, 0) & =h_{2}(t), \\
y(0, .) & =y_{0}
\end{aligned}
$$

satisfies $y(T,)=.y_{T}$.

Notice that explicit controls may be given. Unfortunately, the state $y$ is only known to belong to $C\left([0, T],\left(H_{p}^{2}\right)^{\prime}\right)$ so it seems quite difficult to deduce from Theorem 1.1 controllability results for nonlinear KdV equation (1.1). Lastly it should be more realist to set boundary conditions on the value or the first (spatial) derivative of the function $y$ rather than on the second derivative $y_{x x}$, if we have in mind to test such controllability result or the validity of KdV equation as a model with an experimental device: For instance we may observe the propagation of water waves of small amplitude in a bounded channel, the level -or the slope- of the surface of the fluid being controlled at the ends of the channel. Notice that, as it was suggested in [2], the extra term $y_{x}$ should be in this case incorporated in the equation in order to obtain an appropriate model for water waves in a uniform channel when coordinates $x, y$ are taken with respect to a fixed frame. This inclusion is not without consequence in controllability results: condition on the lenght $L$ of the domain appears. The main result concerning (exact) boundary ESAIM: COCV, MaRCH 1997, VOL.2, PP. 33-55 
controllability for linear $\mathrm{KdV}$ equation (with boundary control on $y_{x}$ at $x=L)$ is the following:

Theorem 1.2. Let $\mathcal{N}=\left\{2 \pi \sqrt{\frac{k^{2}+k l+l^{2}}{3}} ; k, l \in N^{*}\right\}$. Then for any $T>0$ and $L \in(0,+\infty) \backslash \mathcal{N}$, for any $y_{0}, y_{T} \in L^{2}(0, L)$, there exists $h \in L^{2}(0, T)$ such that the mild solution $y \in C\left([0, T], L^{2}(0, L)\right) \cap L^{2}\left(0, T, H^{1}(0, L)\right)$ of

$$
\begin{aligned}
y_{t}+y_{x}+y_{x x x} & =0 \\
y(t, 0)=y(t, L) & =0 \\
y_{x}(t, L) & =h(t) \\
y(0, x) & =y_{0}
\end{aligned}
$$

satisfies $y(T,)=.y_{T}$.

This theorem is proved by means of H.U.M and multiplier method. It turns out that the study of (1.14)-(1.17) as a boundary initial-value problem is more delicate than for (1.9)-(1.13), and that - because of the extra term $y_{x}$ in (1.14) - the (key) observability result holds true iff $L \notin \mathcal{N}$. On the other hand the solution $y$ belongs this time to a functional space in which we may give a sense to the nonlinear term $y y_{x}$ in (1.1).

As a consequence of previous controllability result we get the main result of the paper, that is the exact boundary controllability of nonlinear KdV equation on a bounded domain:

TheOREM 1.3. Let $T>0$ and $L>0$. Then there exists $r_{0}>0$ such that for any $y_{0}, y_{T} \in L^{2}(0, L)$ with $\left\|y_{0}\right\|_{L^{2}(0, L)}<r_{0},\left\|y_{T}\right\|_{L^{2}(0, L)}<r_{0}$, there exists $y \in C\left([0, T], L^{2}(0, L)\right) \cap L^{2}\left(0, T, H^{1}(0, L)\right) \cap W^{1,1}\left(0, T, H^{-2}(0, L)\right)$ solution of

$$
\begin{aligned}
y_{t} & =-\left(y_{x}+y y_{x}+y_{x x x}\right) \text { in } \mathcal{D}^{\prime}\left(0, T, H^{-2}(0, L)\right) \\
y(., 0) & =0 \text { in } L^{2}(0, T),
\end{aligned}
$$

and such that $y(0,)=.y_{0}, y(T,)=.y_{T}$. If moreover $L \notin \mathcal{N}$, then we may in addition assume that $y(., L)=0$ in $L^{2}(0, T)$ and take $y_{x}(., L)$ in $L^{2}(0, T)$ as control function.

Theorem 1.3 is proved by means of Banach contraction fixed point theorem and Theorem 1.2.

The paper is organized as follows: In Section 2 we derive from H.U.M. a direct proof of the exact boundary controllability result by Russell-Zhang for (linear) KdV equation (Th. 1.1). In Section 3 we consider another boundary controllability problem for (linear) KdV equation, in which only the value of first spatial derivative (at $x=L$ ) of the state function is assumed to be controlled: this boundary initial-value problem is first shown to admit solutions (Prop. 3.7). Next an observability result is given (Prop. 3.9) and used to show (applying H.U.M) the exact boundary controllability for linear KdV equation with these boundary conditions (Th. 1.2). Finally in Section 4 we derive from Th. 1.2 the main result of this paper, that is the exact 
(local) boundary controllability of (nonlinear) KdV equation on a bounded domain (Th. 1.3).

\section{EXACT BOUNDARY CONTROLLABILITY OF LINEAR KDV EQUATION BY MEANS OF CONTROL ON DATA

$$
\left[\frac{\partial^{k} y}{\partial x^{k}}(t, .)\right]_{0}^{2 \pi}, k=0,1,2 .
$$

In this section, for reasons of simplicity, we restrict ourselves to the case where the space domain $[0, L]$ is $[0,2 \pi]$, although Theorem 1.1 holds true for arbitrary $L>0$. On the other hand all the functions of this section are assumed to take complex values. For $k \in\{2,3\}$ we set

$$
H_{p}^{k}:=\left\{u \in H^{k}(0,2 \pi) ; \frac{d^{j} u}{d x^{j}}(0)=\frac{d^{j} u}{d x^{j}}(2 \pi) \text { for } 0 \leq j \leq k-1\right\} .
$$

$\left(H^{k}(0,2 \pi)\right.$ denotes classical Sobolev space on the interval $(0,2 \pi)$.) For $n \in$ $\mathbb{Z}$, let

$$
\hat{u}(n)=\frac{1}{2 \pi} \int_{0}^{2 \pi} u(t) e^{-i n t} d t
$$

be the nth Fourier coefficient of $u \in L^{2}(0,2 \pi)$. It is easy to see that, for all $u \in L^{2}(0,2 \pi), k \in\{2,3\}$ :

$$
u \in H_{p}^{k} \Longleftrightarrow \sum_{n \in \mathbb{Z}}\left(n^{k}|\hat{u}(n)|\right)^{2}<\infty
$$

and that the Sobolev norm $\|u\|_{k}:=\left(\sum_{j=0}^{k} \int_{0}^{2 \pi}\left|u^{(j)}(x)\right|^{2} d x\right)^{\frac{1}{2}}$ reduces to

$$
\|u\|_{k}=\left(\sum_{n \in \mathbb{Z}}\left(1+n^{2}+\ldots+n^{2 k}\right)|\hat{u}(n)|^{2}\right)^{\frac{1}{2}}
$$

for $u \in H_{p}^{k}$. In what follows the (Hilbert) space $H_{p}^{k}$ is endowed with the norm $\|\cdot\|_{k}$.

Let $A$ denote the operator $A u=-u^{\prime \prime \prime}$ on the domain $\mathcal{D}(A)=H_{p}^{3} \subset$ $L^{2}(0,2 \pi)$. As it is mentioned in [16], $A$ generates a strongly continuous unitary group $(S(t))_{t \in \mathbb{R}}$ on $L^{2}(0,2 \pi)$. Let $T>0$. For $u_{T}=\sum_{n \in \mathbb{Z}} c_{n} \epsilon^{i n t} \in$ $L^{2}(0,2 \pi)$, the (mild) solution of the uncontrolled problem:

$$
\begin{aligned}
u_{t}+u_{x x x} & =0, \quad x \in(0,2 \pi), t \in \mathbb{R} \\
u(t, 0) & =u(t, 2 \pi), \\
u_{x}(t, 0) & =u_{x}(t, 2 \pi), \\
u_{x x}(t, 0) & =u_{x x}(t, 2 \pi), \\
u(T, .) & =u_{T}
\end{aligned}
$$

is given by

$$
u(t, x)=\sum_{n \in \mathbb{Z}} c_{n} e^{i\left(n^{3}(t-T)+n x\right)} .
$$

(In the following, $u$ will denote the solution of (2.5)-(2.9) associated with $\left.u_{T} \cdot\right)$

Esaim: CoCV, March 1997, Vol.2, PP. 33-55 
We now consider the following non-homogeneous problem: Given $y_{0} \in\left(H_{p}^{2}\right)^{\prime}$, $h_{0}, h_{1}, h_{2}$ in $L^{2}(0, T)$, find $y$ such that:

$$
\begin{aligned}
y_{t}+y_{x x x} & =0, \quad x \in(0,2 \pi), t \in(0, T) \\
y(t, 2 \pi)-y(t, 0) & =h_{0}(t), \\
y_{x}(t, 2 \pi)-y_{x}(t, 0) & =h_{1}(t), \\
y_{x x}(t, 2 \pi)-y_{x x}(t, 0) & =h_{2}(t), \\
y(0, .) & =y_{0} .
\end{aligned}
$$

We first prove that 1) equations (2.11)-(2.15) admit a unique solution $y \in$ $C\left([0, T],\left(H_{p}^{2}\right)^{\prime}\right)$ in a sense to be made precise, and 2$)$ this solution is the classical one whenever $y_{0} \in \mathcal{D}(A)$, and $h_{0}, h_{1}, h_{2}$ are smooth enough and vanish at 0 . More precisely, we prove the following:

Proposition 2.1. 1) Assume $h_{0}, h_{1}, h_{2} \in C_{0}^{2}([0, T]):=\left\{h \in C^{2}\left([0, T], Q^{\prime}\right)\right.$; $h(0)=0\}$ and $y_{0} \in H_{p}^{3}$. Then there exists a unique solution $y \in$ $C\left([0, T], H^{3}(0,2 \pi)\right) \cap C^{1}\left([0, T], L^{2}(0,2 \pi)\right)$ of (2.11)-(2.15). Moreover for any $u_{T} \in H_{p}^{3}$ and any $S \in[0, T]$ we have

$$
\begin{aligned}
& \int_{0}^{2 \pi} u(S, x) \overline{y(S, x)} d x \\
= & \int_{0}^{2 \pi} u(0, x) \overline{y_{0}(x)} d x \\
- & \int_{0}^{S}\left(u_{x x}(t, 0) \overline{h_{0}(t)}-u_{x}(t, 0) \overline{h_{1}(t)}+u(t, 0) \overline{h_{2}(t)}\right) d t .
\end{aligned}
$$

2) For $u_{T} \in H_{p}^{2}, u \in C\left([0, T], H_{p}^{2}\right)$ and $u_{x x}(., 0)$ makes sense in $L^{2}(0, T)$. 3) Assume now only that $y_{0} \in\left(H_{p}^{2}\right)^{\prime}$ and $h_{0}, h_{1}, h_{2} \in L^{2}(0, T)$. Then there exists a unique $y \in C\left([0, T],\left(H_{p}^{2}\right)^{\prime}\right)$ such that

$$
\begin{gathered}
\forall u_{T} \in H_{p}^{2}, \forall S \in[0, T] \\
<y(S), u(S, .)>_{\left(H_{p}^{2}\right)^{\prime}, H_{p}^{2}}=<y_{0}, u(0, .)>_{\left(H_{p}^{2}\right)^{\prime}, H_{p}^{2}} \\
-\int_{0}^{S}\left(u_{x x}(t, 0) \overline{h_{0}(t)}-u_{x}(t, 0) \overline{h_{1}(t)}+u(t, 0) \overline{\left.h_{2}(t)\right)} d t .\right.
\end{gathered}
$$

Proof. 1) Let $\phi_{0}, \phi_{1}, \phi_{2} \in C^{\infty}([0,2 \pi])$ be such that $\phi_{i}^{(k)}(0)=0$ and $\phi_{i}^{(k)}(2 \pi)$ $=-\delta_{i}^{k}$ for $i, k=0,1,2$. The change of function

$$
z(t, x):=h_{0}(t) \phi_{0}(x)+h_{1}(t) \phi_{1}(x)+h_{2}(t) \phi_{2}(x)+\left(S(t) y_{0}\right)(x)+y(t, x)
$$

yields an equivalent problem: Find $z$ such that

$$
\begin{aligned}
z_{t}+z_{x x x} & =f(t, x):=\sum_{i=0}^{2}\left(h_{i}^{\prime}(t) \phi_{i}(x)+h_{i}(t) \phi_{i}^{\prime \prime \prime}(x)\right) \\
z(t, 2 \pi) & =z(t, 0) \\
z_{x}(t, 2 \pi) & =z_{x}(t, 0) \\
z_{x x}(t, 2 \pi) & =z_{x x}(t, 0) \\
z(0, .) & =0 .
\end{aligned}
$$


Since $f \in C^{1}\left([0, T], L^{2}(0,2 \pi)\right)$, this non-homogeneous problem admits (see [15]) a unique solution $z \in C\left([0, T], H_{p}^{3}\right) \cap C^{1}\left([0, T], L^{2}(0,2 \pi)\right)$. This proves the first assertion in 1$)$. Let $u_{T} \in H_{p}^{3}$. Then $u \in C\left([0, T], H_{p}^{3}\right) \cap$ $C^{1}\left([0, T], L^{2}(0,2 \pi)\right)$. Integrations by parts in

$$
\int_{0}^{S} \int_{0}^{2 \pi}\left(u_{t}+u_{x x x}\right) \bar{y} d x d t=0
$$

lead to

$$
\begin{aligned}
& -\int_{0}^{S} \int_{0}^{2 \pi} u\left(\overline{y_{t}}+\overline{y_{x x x}}\right) d x d t+\int_{0}^{2 \pi}[(u \bar{y})(., x)]_{0}^{S} d x \\
& +\int_{0}^{S}\left[\left(u_{x x} \bar{y}-u_{x} \overline{y_{x}}+u \overline{y_{x x}}\right)(t, .)\right]_{0}^{2 \pi} d t=0 .
\end{aligned}
$$

Taking into account (2.6)-(2.8) and (2.11)-(2.15), we get (2.16).

2) By (2.10), for $t_{1}, t_{2} \in[0, T]$

$$
u\left(t_{1}, x\right)-u\left(t_{2}, x\right)=\sum_{n \in \mathbb{Z}} c_{n} e^{-i n^{3} T}\left(e^{i n^{3} t_{1}}-e^{i n^{3} t_{2}}\right) e^{i n x} .
$$

If $u_{T} \in H_{p}^{2}, \sum_{n \in \mathbb{Z}}\left|n^{2} c_{n}\right|^{2}<\infty$ and it follows from Lebesgue's theorem that $\sum_{n \in \mathbb{Z}}\left|n^{2} c_{n} e^{-i n^{3} T}\left(e^{i n^{3} t_{1}}-e^{i n^{3} t_{2}}\right)\right|^{2} \rightarrow 0$ as $t_{1} \rightarrow t_{2}$. Then $u \in C\left([0, T], H_{p}^{2}\right)$. Hence $u(., 0)$ and $u_{x}(., 0)$ exist in $C([0, T]) \subset L^{2}(0, T)$. The same argument shows that if $u_{T} \in H_{p}^{3}, u \in C\left([0, T], H_{p}^{3}\right)$ and

$$
u_{x x}(t, 0)=\sum_{n \in \mathbb{Z}}\left(-n^{2} e^{-i n^{3} T} c_{n}\right) e^{i n^{3} t} .
$$

The sum in $(2.27)$ makes sense in $L^{2}(0, T)$ whenever $\sum_{n \in \mathbb{Z}}\left(n^{2}\left|c_{n}\right|\right)^{2}<\infty$, that is $u_{T} \in H_{p}^{2}$. From now on $u_{x x}(., 0)$ denotes, for $u_{T} \in H_{p}^{2}$, the sum in (2.27). Notice that the linear map $u_{T} \mapsto u_{x x}(., 0)$ is continuous since

$$
\left\|\sum_{n \in \mathbb{Z}}\left(-n^{2} e^{-i n^{3} T} c_{n}\right) e^{i n^{3} t}\right\|_{L^{2}(0, T)}^{2} \leq\left(\left[\frac{T}{2 \pi}\right]+1\right) \sum_{n \in \mathbb{Z}}\left(n^{2}\left|c_{n}\right|\right)^{2},
$$

where $[x]$ denotes the integral part of a real number $x$.

3 ) Identifying $L^{2}(0,2 \pi)$ with its dual by means of the (conjugate linear) map $y \mapsto(., y)_{L^{2}(0,2 \pi)}$, we have the following diagram:

$$
H_{p}^{2} \subset L^{2}(0,2 \pi)=L^{2}(0,2 \pi)^{\prime} \subset\left(H_{p}^{2}\right)^{\prime}
$$

where each embedding is dense and compact. Moreover

$$
<y, u>_{\left(H_{p}^{2}\right)^{\prime}, H_{p}^{2}}=(u, y)_{L^{2}(0,2 \pi)}\left(=\int_{0}^{2 \pi} u \bar{y} d x\right)
$$

for $u \in H_{p}^{2}$ and $y \in L^{2}(0,2 \pi)$. It follows that (2.17) holds true for $h_{0}, h_{1}, h_{2}$ $\in C_{0}^{2}([0, T])$ and $y_{0}, u_{T} \in H_{p}^{3}$. Since $H_{p}^{3}$ is dense in $H_{p}^{2}$, using 2$)$ we see that (2.17) also be true for $u_{T} \in H_{p}^{2}$. For $y_{0} \in\left(H_{p}^{2}\right)^{\prime}$ and $h_{0}, h_{1}, h_{2} \in L^{2}(0, T)$, we define a "weak" solution of (2.11)-(2.15) as a function $y \in C\left([0, T],\left(H_{p}^{2}\right)^{\prime}\right)$ such that (2.17) holds true for all $u_{T} \in H_{p}^{2}$ and all $S \in[0, T]$. Let $S \in[0, T]$ Esaim: CoCV, MARCH 1997, Vol.2, PP. 33-55 
be fixed. We first show that $(2.17)$ defines $y(S)$ in $\left(H_{p}^{2}\right)^{\prime}$ in a unique manner. It follows from the proof of 2 ) that the map

$$
u_{T} \in H_{p}^{2} \mapsto-\int_{0}^{S}\left(u_{x x}(t, 0) \overline{h_{0}(t)}-u_{x}(t, 0) \overline{h_{1}(t)}+u(t, 0) \overline{h_{2}(t)}\right) d t \in Q^{\prime}
$$

is a continuous linear form. On the other hand (see (2.3), (2.4) and (2.10)) the map $u_{T} \in H_{p}^{2} \mapsto u(S,.) \in H_{p}^{2}$ is clearly an automorphism of Hilbert space. Hence for each $S \in[0, T], y(S)$ is uniquely defined in $\left(H_{p}^{2}\right)^{\prime}$. Moreover for $S \in[0, T]$,

$$
\begin{aligned}
& \|y(S)\|_{\left(H_{p}^{2}\right)^{\prime}} \\
= & \sup _{\|u(S, .)\|_{2} \leq 1}|<y(S), u(S, .)>| \\
= & \sup _{\left\|u_{T}\right\|_{2} \leq 1} \mid<y_{0}, u(0, .)>\left(H_{p}^{2}\right)^{\prime}, H_{p}^{2} \\
& \quad-\int_{0}^{S}\left(u_{x x}(t, 0) \overline{h_{0}(t)}-u_{x}(t, 0) \overline{h_{1}(t)}+u(t, 0) \overline{h_{2}(t)}\right) d t \mid \\
& \quad \sup _{\left\|u_{T}\right\|_{2} \leq 1}\left(\left\|y_{0}\right\|_{\left(H_{p}^{2}\right)^{\prime}} \cdot\|u(0, .)\|_{H_{p}^{2}}+\left\|u_{x x}(., 0)\right\|_{L^{2}(0, T)} \cdot\left\|h_{0}\right\|_{L^{2}(0, T)}\right. \\
& \left.+\left\|u_{x}(., 0)\right\|_{L^{2}(0, T)} \cdot\left\|h_{1}\right\|_{L^{2}(0, T)}+\|u(., 0)\|_{L^{2}(0, T)} \cdot\left\|h_{2}\right\|_{L^{2}(0, T)}\right) \\
\leq \quad & C\left(\left\|y_{0}\right\|_{\left(H_{p}^{2}\right)^{\prime}}+\left\|h_{0}\right\|_{L^{2}(0, T)}+\left\|h_{1}\right\|_{L^{2}(0, T)}+\left\|h_{2}\right\|_{L^{2}(0, T)}\right),
\end{aligned}
$$

where $C$ is a positive constant which does not depend on $S$ or on $y_{0}, h_{0}, h_{1}$, $h_{2}$. Since

$$
y \in C\left([0, T], L^{2}(0,2 \pi)\right) \subset C\left([0, T],\left(H_{p}^{2}\right)^{\prime}\right)
$$

for $y_{0} \in H_{p}^{3}$ and $\left(h_{0}, h_{1}, h_{2}\right) \in C_{0}^{2}([0, T])^{3}$, and since $H_{p}^{3}$ is dense in $L^{2}(0, L)$ and $C_{0}^{2}([0, T])$ is dense in $L^{2}(0, T)$, it follows from (2.32) that $y \in$ $C\left([0, T],\left(H_{p}^{2}\right)^{\prime}\right)$ in the general case too.

For applying H.U.M. we need the following (key) observability result.

Proposition 2.2. Let $T>0$. Then there exist positive numbers $C_{1}^{T}, C_{2}^{T}$ such that for every $u_{T} \in H_{p}^{2}$

$$
\begin{aligned}
C_{1}^{T}\left\|u_{T}\right\|_{2}^{2} & \leq\|u(., 0)\|_{L^{2}(0, T)}^{2}+\left\|u_{x}(., 0)\right\|_{L^{2}(0, T)}^{2}+\left\|u_{x x}(., 0)\right\|_{L^{2}(0, T)}^{2} \\
& \leq C_{2}^{T}\left\|u_{T}\right\|_{2}^{2} .
\end{aligned}
$$

Proof. We have in $L^{2}(0, T)$ :

$$
\begin{aligned}
u(t, 0) & =\sum_{n \in \mathbb{Z}} c_{n} e^{i n^{3}(t-T)}, \\
u_{x}(t, 0) & =\sum_{n \in \mathbb{Z}} i n c_{n} e^{i n^{3}(t-T)}, \\
u_{x x}(t, 0) & =\sum_{n \in \mathbb{Z}}-n^{2} c_{n} e^{i n^{3}(t-T)} .
\end{aligned}
$$


Hence

$$
\begin{aligned}
& \|u(., 0)\|_{L^{2}(0, T)}^{2}+\left\|u_{x}(., 0)\right\|_{L^{2}(0, T)}^{2}+\left\|u_{x x}(., 0)\right\|_{L^{2}(0, T)}^{2} \\
\leq & \left(\left[\frac{T}{2 \pi}\right]+1\right) \sum_{n \in \mathbb{Z}}\left(1+n^{2}+n^{4}\right)\left|c_{n}\right|^{2} \\
\leq & C_{2}^{T}\left\|u_{T}\right\|_{2}^{2} .
\end{aligned}
$$

if we set $C_{2}^{T}:=\left[\frac{T}{2 \pi}\right]+1$. To prove the left inequality we first take $T^{\prime} \in(0, T)$ and $\gamma>\frac{2 \pi}{T^{\prime}}$.

Let $N \in I N^{*}$ be such that

$$
n \in \mathbb{Z},|n| \geq N \Rightarrow(n+1)^{3}-n^{3} \geq \gamma
$$

By Ingham's inequality (see [6]) there exists $C^{T^{\prime}}>0$ such that

$$
\forall\left(a_{n}\right)_{n \in \mathbb{Z}} \in l^{2}(\mathbb{Z}) \quad \sum_{|n| \geq N}\left|a_{n}\right|^{2} \leq C^{T^{\prime}} \int_{0}^{T^{\prime}}\left|\sum_{|n| \geq N} a_{n} e^{i n^{3}(t-T)}\right|^{2} d t .
$$

Let $\mathcal{Z}_{n}:=\operatorname{Span}\left(e^{i n x}\right)$ for $n \in \mathbb{Z}$ and $\mathcal{Z}:=\oplus_{n \in \mathbb{Z}_{n}} \subset \mathcal{Z}_{p}^{2}$. We define a semi-norm $p$ in $\mathcal{Z}$ by

$\forall u \in \mathcal{Z}$

$$
\begin{aligned}
p(u) & =\left(|u(0)|^{2}+\left|u^{\prime}(0)\right|^{2}+\left|u^{\prime \prime}(0)\right|^{2}\right)^{\frac{1}{2}} \\
& =\left(\left|\sum_{n \in \mathbb{Z}} \hat{u}(n)\right|^{2}+\left|\sum_{n \in \mathbb{Z}} i n \hat{u}(n)\right|^{2}+\left|\sum_{n \in \mathbb{Z}}-n^{2} \hat{u}(n)\right|^{2}\right)^{\frac{1}{2}}
\end{aligned}
$$

(For $u \in \mathcal{Z}, \hat{u}(n)=0$ for $|n|$ large enough.) Let $u_{T} \in \mathcal{Z} \cap\left(\oplus_{|n|<N} \mathcal{Z}_{n}\right)^{\perp}$, that is $c_{n}=0$ for $|n|<N$ or for $|n|$ large enough. Using (2.10), (2.40) we get

$$
\left\|u_{T}\right\|_{2}^{2}=\sum_{|n| \geq N}\left(1+n^{2}+n^{4}\right)\left|c_{n}\right|^{2} \leq C^{T^{\prime}} \int_{0}^{T^{\prime}} p(u(t, .))^{2} d t .
$$

Since $T>T^{\prime}$, it follows from $(2.38),(2.43)$ and a result by Komornik (see [7; Th. 5.2]) that there exists a constant $C_{1}^{T}>0$ such that

$$
\begin{aligned}
\forall u_{T} \in \mathcal{Z} \quad C_{1}^{T}\left\|u_{T}\right\|_{2}^{2} & \leq \int_{0}^{T} p(u(t, .))^{2} d t \\
& =\|u(., 0)\|_{L^{2}(0, T)}^{2}+\left\|u_{x}(., 0)\right\|_{L^{2}(0, T)}^{2} \\
& +\left\|u_{x x}(., 0)\right\|_{L^{2}(0, T)}^{2}
\end{aligned}
$$

We get the left inequality (in (2.34)) in the general case $\left(u_{T} \in H_{p}^{2}\right)$ by a density argument.

We may now complete the proof of Theorem 1.1 by applying H.U.M.

Proof of Theorem 1.1. We first remark that we may assume $y_{0}=0$. Indeed if $y_{0}, y_{T}$ are given in $\left(H_{p}^{2}\right)^{\prime}$, if there exist $h_{0}, h_{1}, h_{2} \in L^{2}(0, T)$ such that the (weak) solution $\tilde{y}$ of $(2.11)-(2.14)$ and $\tilde{y}(0,)=$.0 satisfies $\tilde{y}(T,)=.y_{T}-$ Esaim: COCV, MaRCH 1997, VOL.2, PP. 33-55 
$S(T) y_{0}$, then $y(t,):.=S(t) y_{0}+\tilde{y}(t,$.$) is the (weak) solution of (2.11)-(2.15)$ (with the same control functions) and is such that $y(T,)=.y_{T}$. In what follows we assume $y_{0}=0$. For $u_{T} \in H_{p}^{2}$ we let $\Lambda\left(u_{T}\right)$ denote $y(T) \in\left(H_{p}^{2}\right)^{\prime}$, where $y$ is the (weak) solution of (2.11)-(2.15) and $h_{0}, h_{1}$ and $h_{2}$ are chosen in the following way:

$$
\begin{aligned}
& h_{0}(t):=-u_{x x}(t, 0) \\
& h_{1}(t):=u_{x}(t, 0) \\
& h_{2}(t):=-u(t, 0) .
\end{aligned}
$$

(As a bove 1 stands for the solution of (2.5)-(2.9) associated with $u_{T}$.) Clearly $\Lambda: H_{p}^{2} \rightarrow\left(H_{p}^{2}\right)^{\prime}$ is a (conjugate linear) continuous map. Moreover

$$
\begin{aligned}
& <\Lambda\left(u_{T}\right), u_{T}>\left(H_{p}^{2}\right)^{\prime}, H_{p}^{2} \\
= & \int_{0}^{T}\left(|u(t, 0)|^{2}+\left|u_{x}(t, 0)\right|^{2}+\left|u_{x x}(t, 0)\right|^{2}\right) d t \\
\geq & C_{1}^{T}\left\|u_{T}\right\|_{2}^{2}
\end{aligned}
$$

by Propositions 2.1 and 2.2. It follows from Lax-Milgram theorem (see [19; p.92]) that $\Lambda$ is invertible. The proof of Theorem 1.1 is complete.

REMARK 2.3. (i) If $T=2 \pi$ the observability result is obvious. Indeed for any $u_{T} \in H_{p}^{2}$

$$
\left\|u_{T}\right\|_{2}^{2}=\|u(., 0)\|_{L^{2}(0,2 \pi)}^{2}+\left\|u_{x}(., 0)\right\|_{L^{2}(0,2 \pi)}^{2}+\left\|u_{x x}(., 0)\right\|_{L^{2}(0,2 \pi)}^{2} .
$$

(ii) Setting $h_{1}(t)=0$ instead of $u_{x}(t, 0)$ we also get (for some constant $\left.\bar{C}_{1}^{T}>0\right)$

$$
\forall u_{T} \in H_{p}^{2}<\Lambda\left(u_{T}\right), u_{T}>_{\left(H_{p}^{2}\right)^{\prime}, H_{p}^{2}} \geq \bar{C}_{1}^{T}\left\|u_{T}\right\|_{2}^{2},
$$

so $\Lambda$ is also onto.

(iii) If we assume $h_{0}(t)=h_{2}(t)=0$, the same arguments as above yield the following result: for every $T>0$ and $y_{0}, y_{T} \in\left(H_{p}^{1}\right)^{\prime}$ there exists $h_{1} \in$ $L^{2}(0, T)$ such that the solution $y \in C\left([0, T],\left(H_{p}^{1}\right)^{\prime}\right)$ of $(1.9)-(1.13)$ (with $\left.h_{0}=h_{2}=0\right)$ satisfies $y(T,)=.y_{T}$. Indeed it may be shown that for $u_{T} \in H_{p}^{1}$ $u_{x}(., 0)$ makes sense in $L^{2}(0, T)$ and that $C_{3}^{T}\left\|u_{T}\right\|_{1}^{2} \leq\left\|u_{x}(., 0)\right\|_{L^{2}(0, T)}^{2} \leq$ $C_{4}^{T}\left\|u_{T}\right\|_{1}^{2}$ for some constants $C_{3}^{T}, C_{4}^{T}>0$. When we compare these results with those by Russell-Zhang, we remark that 1$)$ the spaces in which we get exact controllability are larger than $\left.L^{2}(0, L)\left(L^{2}(0, L) \subset\left(H_{p}^{1}\right)^{\prime} \subset\left(H_{p}^{2}\right)^{\prime}\right) ; 2\right)$ the assumption (1.8) is not used here and 3 ) the control may be explicitly given : for reaching $y_{T} \in\left(H_{p}^{2}\right)^{\prime}$ we may choose $h_{0}, h_{1}, h_{2}$ given by (2.45)(2.47), with $u_{T}=\Lambda^{-1}\left(y_{T}-S(T) y_{0}\right)$. However, even if $y_{0}, y_{T} \in L^{2}(0,2 \pi), y$ is only known to belong to $C\left([0, T],\left(H_{p}^{2}\right)^{\prime}\right)$. So this method is not appropriate for starting the study of controllability of nonlinear KdV equation. In the following section, another (more appropriate) exact controllability result for linear KdV equation with different boundary conditions is given. 


\section{EXACT BOUNDARY CONTROLLABILITY OF LINEAR KDV EQUATION BY MEANS OF CONTROL ON $y_{x}(t, L)$.}

From now on, the scalar space is $I R$. In this section $L$ stands for some positive number. We shall prove the controllability in $L^{2}(0, L)$ of

$$
\begin{aligned}
y_{t}+y_{x}+y_{x x x} & =0 \\
y(t, 0)=y(t, L) & =0 \\
y_{x}(t, L) & =h(t) \\
y(0, x) & =y_{0}
\end{aligned}
$$

where $h \in L^{2}(0, T)$ stands for the control function. More precisely we shall prove that, for any $L>0, T>0$, for any $y_{0}, y_{T} \in L^{2}(0, L)$ there exists $h \in L^{2}(0, T)$ such that a mild solution $y \in C\left([0, T], L^{2}(0, L)\right) \cap$ $L^{2}\left(0, T, H^{1}(0, L)\right) \cap H^{1}\left(0, T, H^{-2}(0, L)\right)$ of (3.1)-(3.4) (which verifies (3.1) in $\mathcal{D}^{\prime}\left(0, T, H^{-2}\right)$ and $(3.4)$ in $\left.L^{2}(0, L)\right)$, may be found such that $y(T,)=.y_{T}$.

We begin by showing the well-posedness of the (initial-value) homogeneous problem:

$$
\begin{aligned}
y_{t}+y_{x}+y_{x x x} & =0 \\
y(t, 0)=y(t, L) & =0 \\
y_{x}(t, L) & =0 \\
y(0, x) & =y_{0}(x) .
\end{aligned}
$$

Let $A$ denote the operator $A w=-w^{\prime \prime \prime}-w^{\prime}$ on the (dense) domain $\mathcal{D}(A) \subset$ $L^{2}(0, L)$ defined by

$$
\mathcal{D}(A)=\left\{w \in H^{3}(0, L) ; w(0)=w(L)=w^{\prime}(L)=0\right\} .
$$

The following result holds:

Proposition 3.1. A generates a strongly continuous semigroup of contractions on $L^{2}(0, L)$.

Proof. It is easy to see that $A$ is closed. Let $w \in \mathcal{D}(A)$. Then

$$
\begin{aligned}
(w, A w)_{L^{2}(0, L)} & =\int_{0}^{L} w(x)\left(-w^{\prime \prime \prime}(x)-w^{\prime}(x)\right) d x \\
& =\int_{0}^{L} w^{\prime}(x) w^{\prime \prime}(x) d x-\left[w w^{\prime \prime}\right]_{0}^{L}-\left[\frac{w^{2}}{2}\right]_{0}^{L} \\
& =\left[\frac{w^{\prime 2}}{2}\right]_{0}^{L} \\
& =-\frac{w^{\prime}(0)^{2}}{2} \leq 0 .
\end{aligned}
$$

Hence $A$ is dissipative. It may be seen that $A^{*}(w)=w^{\prime}+w^{\prime \prime \prime}$ with domain $\mathcal{D}\left(A^{*}\right)=\left\{w \in H^{3}(0, L) ; w(0)=w(L)=w^{\prime}(0)=0\right\}$, so that $\left(w, A^{*}(w)\right)=-\frac{w^{\prime}(L)^{2}}{2} \leq 0$ and $A^{*}$ is dissipative too. Now the result follows from $[15$; cor. 4.4 chapter 1$]$.

From now on we let $\left(S(t)_{t>0}\right)$ denote the semi-group of contractions associated with $A$, and we let $B$ denote the (Banach) space $C\left([0, T], L^{2}(0, L)\right) \cap$ ESAIM: COCV, MaRCH 1997, VOL.2, PP. 33-55 
$L^{2}\left(0, T, H^{1}(0, L)\right)$ endowed with the norm

$$
\|y\|_{B}:=\sup _{t \in[0, T]}\|y(t, .)\|_{L^{2}(0, L)}+\left(\int_{0}^{T}\|y(t, .)\|_{H^{1}(0, L)}^{2} d t\right)^{\frac{1}{2}} .
$$

Using the multiplier method, we get useful estimates for the mild solutions of (3.5)-(3.8).

Proposition 3.2. 1) The map $y_{0} \in L^{2}(0, L) \mapsto S(.) y_{0} \in B$ is continuous. 2) For $y_{0} \in L^{2}(0, L), y_{x}(., 0)$ makes sense in $L^{2}(0, L)$, and

$$
\begin{aligned}
& \forall y_{0} \in L^{2}(0, L) \\
& \left\|y_{x}(., 0)\right\|_{L^{2}(0, T)} \leq\left\|y_{0}\right\|_{L^{2}(0, L)}, \\
& \left\|y_{0}\right\|_{L^{2}(0, L)}^{2} \leq \frac{1}{T}\left\|S(\cdot) y_{0}\right\|_{L^{2}((0, T) \times(0, L))}^{2}+\left\|y_{x}(\cdot, 0)\right\|_{L^{2}(0, T)}^{2} .
\end{aligned}
$$

Proof. 1) For $y_{0} \in L^{2}(0, L)$ we write $y$ the mild solution $S(\cdot) y_{0}$ of (3.5)-(3.8). By Proposition $3.1 y \in C\left([0, T], L^{2}(0, L)\right)$ and

$$
\|y\|_{C\left([0, T], L^{2}(0, L)\right)} \leq\left\|y_{0}\right\|_{L^{2}(0, L)} .
$$

To see that $y \in L^{2}\left(0, T, H^{1}(0, L)\right)$ we first assume $y \in \mathcal{D}(A)$. Let $q \in$ $C^{\infty}([0, T] \times[0, L])$. Integrating by parts in

$$
\int_{0}^{T} \int_{0}^{L} q y\left(y_{t}+y_{x}+y_{x x x}\right) d x d t=0
$$

using (3.5)-(3.8) (and Fubini's theorem) we get:

$$
\begin{aligned}
& -\int_{0}^{T} \int_{0}^{L}\left(q_{t}+q_{x}+q_{x x x}\right) \frac{y^{2}}{2} d x d t+\int_{0}^{L}\left(q \frac{y^{2}}{2}\right)(T, x) d x \\
& -\quad \int_{0}^{L} q(0, x) \frac{y_{0}^{2}(x)}{2} d x+\frac{3}{2} \int_{0}^{T} \int_{0}^{L} q_{x} y_{x}^{2} d x d t \\
& +\quad \int_{0}^{T}\left(q \frac{y_{x}^{2}}{2}\right)(t, 0) d t=0 .
\end{aligned}
$$

Choosing $q(t, x)=x$ leads to

$$
\begin{array}{r}
-\int_{0}^{T} \int_{0}^{L} y^{2} d x d t+\int_{0}^{L} x y(T, x)^{2} d x-\int_{0}^{L} x y_{0}(x)^{2} d x \\
+3 \int_{0}^{T} \int_{0}^{L} y_{x}^{2} d x d t=0 .
\end{array}
$$

Hence

$$
\int_{0}^{T} \int_{0}^{L} y_{x}^{2} d x d t \leq \frac{1}{3}\left(\int_{0}^{T} \int_{0}^{L} y^{2} d x d t+L \int_{0}^{L} y_{0}(x)^{2} d x\right)
$$

and then, using (3.14),

$$
\|y\|_{L^{2}\left(0, T, H^{1}(0, L)\right)} \leq\left(\frac{4 T+L}{3}\right)^{\frac{1}{2}}\left\|y_{0}\right\|_{L^{2}(0, L)} .
$$


By the density of $\mathcal{D}(A)$ in $L^{2}(0, L)$ the result extends to arbitrary $y_{0} \in$ $L^{2}(0, L)$.

2) We also assume $y_{0} \in \mathcal{D}(A)$ and take $q=1$ in (3.16). We get

$$
\int_{0}^{T} y_{x}^{2}(t, 0) d t=\int_{0}^{L} y_{0}^{2}(x) d x-\int_{0}^{L} y^{2}(T, x) d x \leq \int_{0}^{L} y_{0}^{2}(x) d x .
$$

On the other hand the choice $q(t, x)=T-t$ yields

$$
\int_{0}^{T} \int_{0}^{L} y^{2} d x d t-\int_{0}^{L} T y_{0}^{2}(x) d x+\int_{0}^{T}(T-t) y_{x}^{2}(t, 0) d t=0 .
$$

Hence

$$
\int_{0}^{L} y_{0}^{2}(x) d x \leq \frac{1}{T} \int_{0}^{T} \int_{0}^{L} y^{2} d x d t+\int_{0}^{T} y_{x}^{2}(t, 0) d t .
$$

Thanks to (3.20) there exists a unique continuous (linear) extension of the map $y_{0} \in \mathcal{D}(A) \mapsto y_{x}(., 0) \in L^{2}(0, T)$ to the whole space $L^{2}(0, L)$. In the following we also will denote by $y_{x}(., 0)$ the value of this map at any $y_{0} \in$ $L^{2}(0, L)$. Obviously $(3.20)$ and $(3.22)$ hold true for any $y_{0} \in L^{2}(0, L)$.

We now prove the following observability result.

Proposition 3.3. Let $\mathcal{N}=\left\{2 \pi \sqrt{\frac{k^{2}+k l+l^{2}}{3}} ; k, l \in N^{*}\right\}$. Then $\forall L \in$ $(0,+\infty) \backslash \mathcal{N}, \forall T>0, \exists C=C(L, T)>0$ such that

$$
\forall y_{0} \in L^{2}(0, L) \quad\left\|y_{0}\right\|_{L^{2}(0, L)} \leq C\left\|y_{x}(., 0)\right\|_{L^{2}(0, T)} .
$$

Proof. We proceed as in [12: Appendix I] (See also [1]). If the statement is false, there exists a sequence $\left(y_{0}^{n}\right)_{n \geq 0}$ in $L^{2}(0, L)$ such that $\left\|y_{0}^{n}\right\|_{L^{2}(0, L)}=1$ for any $n$ but $\left\|y_{x}^{n}(., 0)\right\|_{L^{2}(0, T)} \rightarrow 0$ as $n \rightarrow \infty$, where $y^{n}:=S(\cdot) y_{0}^{n}$. Clearly $y^{n}$ is bounded in $L^{2}\left(0, T, H^{1}(0, L)\right.$ ) (see (3.19)). On the other hand $y_{t}^{n}=$ $-\left(y_{x}^{n}+y_{x x x}^{n}\right)$ is bounded in $L^{2}\left(0, T, H^{-2}(0, L)\right)$. Since the first embedding in

$$
H^{1}(0, L) \hookrightarrow L^{2}(0, L) \hookrightarrow H^{-2}(0, L)
$$

is compact, it follows from a result by Simon (see [18; cor. 4]) that the set $\left\{y_{n}\right\}$ is relatively compact in $L^{2}\left(0, T, L^{2}(0, L)\right)$. Without loss of generality we may assume that the sequence $\left(y^{n}\right)$ is convergent in $L^{2}\left(0, T, L^{2}(0, L)\right)$. We infer from (3.13) that $\left(y_{0}^{n}\right)$ is a Cauchy sequence in $L^{2}(0, T)$. Let $y_{0}=\lim _{n \rightarrow \infty} y_{0}^{n}$ and $y=S(\cdot) y_{0}$. By Proposition $3.2 y_{x}^{n}(., 0) \rightarrow y_{x}(., 0)$ in $L^{2}(0, T)$. Thus $\left\|y_{0}\right\|_{L^{2}(0, L)}=1$ and $y_{x}(., 0)=0$, but such a function does not exist because of the following:

Lemma 3.4. For $T>0$ let $N_{T}$ denote the space of the (initial) states $y_{0} \in L^{2}(0, L)$ such that the mild solution $y=S(\cdot) y_{0}$ of (3.5)-(3.8) satisfies $y_{x}(\cdot, 0)=0$ in $L^{2}(0, T)$. Then for $L \in(0,+\infty) \backslash \mathcal{N}, N_{T}=(0) \forall T>0$. Proof. Obviously $T<T^{\prime} \Rightarrow N_{T^{\prime}} \subset N_{T}$. On the other hand for any $T>0$ $N_{T}$ is a finite-dimensional vector space: Indeed if $\left(y_{0}^{n}\right)$ is a sequence in the unit ball $\left\{y \in N_{T} ;\|y\|_{L^{2}(0, L)} \leq 1\right\}$ the same argument as above shows that there exists a convergent subsequence. Since the unit ball is compact, $N_{T}$ is finite dimensional by Riesz theorem. Let $T^{\prime}>0$ be given. To prove $N_{T^{\prime}}=(0)$, it is sufficient to find $0<T<T^{\prime}$ such that $N_{T}=(0)$. Since the map $T \mapsto \operatorname{dim}\left(N_{T}\right) \in I N$ is nonincreasing, there exist $T, \epsilon>0$ such that Esaim: COCV, MaRCH 1997 , VOL.2, PP. 33-55 
$T<T+\epsilon<T^{\prime}$ and $\operatorname{dim} N_{T}=\operatorname{dim} N_{T+\epsilon}$. (Hence $N_{t}=N_{T}$ for $T \leq t \leq T+\epsilon$.) Let $y_{0} \in N_{T}, y=S(\cdot) y_{0}$ and $0<t<\epsilon$. Since $S(\tau)\left(S(t) y_{0}\right)=S(\tau+t) y_{0}$ for $0 \leq \tau \leq T$ and $y_{0} \in N_{T+\epsilon}$, we see that

$$
\frac{S(t) y_{0}-y_{0}}{t} \in N_{T} \text {. }
$$

Let

$$
M_{T}:=\left\{\tilde{y}=S(\tau) \tilde{y}_{0} ; 0 \leq \tau \leq T, \tilde{y}_{0} \in N_{T}\right\} \subset C\left([0, T], L^{2}(0, L)\right) .
$$

Since $y \in H^{1}\left(0, T+\epsilon, H^{-2}(0, L)\right)$,

$$
\lim _{t \rightarrow 0^{+}} \frac{y(t+\cdot)-y}{t}=y^{\prime} \text { in } L^{2}\left(0, T, H^{-2}(0, L)\right) .
$$

On the other hand (thanks to (3.25)) $\frac{y(t+\cdot)-y}{t} \in M_{T}$ for $0<t<\epsilon$ and $M_{T}$ is closed in $L^{2}\left(0, T, H^{-2}(0, L)\right.$ ) (since $\left.\operatorname{dim}\left(M_{T}\right)<\infty\right)$. It follows that $y^{\prime} \in C\left([0, T], L^{2}(0, L)\right)$ and $y \in C^{1}\left([0, T], L^{2}(0, L)\right)$, hence we may write

$$
y^{\prime}(0)=\lim _{t \rightarrow 0^{+}} \frac{S(t) y_{0}-y_{0}}{t} \text { in } L^{2}(0, L) .
$$

This in turn implies

$$
y_{0} \in \mathcal{D}(A), \quad A\left(y_{0}\right)=y^{\prime}(0) \in N_{T} \text { and } y_{x}(., 0) \in C([0, T]) .
$$

Thus

$$
\left(\frac{d y_{0}}{d x}\right)_{\mid x=0}=y_{x}(0,0)=0 .
$$

If $N_{T} \neq(0)$, the map $y_{0} \in d N_{T} \mapsto A\left(y_{0}\right) \in G N_{T}$ (where $q^{\prime} N_{T}$ denote the complexification of $N_{T}$ ) has (at least) one eigenvalue, hence there exist $\lambda \in G, y_{0} \in H^{3}(0, L) \backslash\{0\}$ such that (prime denoting here spatial derivative)

$$
\begin{aligned}
& \lambda y_{0}=-y_{0}{ }^{\prime}-y_{0}{ }^{\prime \prime \prime} \\
& y_{0}(0)=y_{0}(L)=y_{0}^{\prime}(0)=y_{0}^{\prime}(L)=0 .
\end{aligned}
$$

We prove in the following lemma that this does not hold if $L \notin \mathcal{N}$.

Lemma 3.5. Let $L \in(0,+\infty)$. Consider the following assertion:

$$
\text { (A) } \exists \lambda \in Q^{\prime}, \exists y_{0} \in H^{3}(0, L) \backslash\{0\} \quad \text { s.t. }\left\{\begin{array}{l}
\lambda y_{0}+y_{0}{ }^{\prime}+y_{0}{ }^{\prime \prime \prime}=0, \\
y_{0}(0)=y_{0}(L)=y_{0}^{\prime}(0) \\
=y_{0}^{\prime}(L)=0 .
\end{array}\right.
$$

Then $(\mathcal{A}) \Longleftrightarrow L \in \mathcal{N}$.

Proof. Assume $y_{0}$ is as in the assertion $(\mathcal{A})$, let us denote by $u \in H^{2}(I R)$ its prolongation by 0 . Then

$$
\lambda u+u^{\prime}+u^{\prime \prime \prime}=y_{0}^{\prime \prime}(0) \delta_{0}-y_{0}^{\prime \prime}(L) \delta_{L} \text { in } \mathcal{D}^{\prime}(I R),
$$

where $\delta_{x_{0}}$ denotes the Dirac measure at $x_{0}$. It is easy to see that $(\mathcal{A})$ is equivalent to the existence of complex numbers $\alpha, \beta, \lambda$ (with $(\alpha, \beta) \neq(0,0)$ ) and of a function $u \in H^{2}(\mathbb{R})$ with compact support in $[-L, L]$ such that

$$
\lambda u+u^{\prime}+u^{\prime \prime \prime}=\alpha \delta_{0}-\beta \delta_{L} \text { in } \mathcal{D}^{\prime}(I R) .
$$


Taking Fourier transform we get

$$
\left(\lambda+(i \xi)+(i \xi)^{3}\right) \hat{u}(\xi)=\alpha-\beta e^{-i L \xi}
$$

hence (setting $\lambda=-i p$ )

$$
\hat{u}(\xi)=i \frac{\alpha-\beta e^{-i L \xi}}{\xi^{3}-\xi+p} .
$$

Using Paley-Wiener theorem (see [19]) and the usual characterization of $H^{2}(\mathbb{R})$ functions by means of their Fourier transforms, we see that $(\mathcal{A})$ is equivalent to the existence of $p \in Q^{\prime}$ and of $(\alpha, \beta) \in Q^{2} \backslash\{(0,0)\}$ such that the map

$$
f(\xi):=\frac{\alpha-\beta e^{-i L \xi}}{\xi^{3}-\xi+p}
$$

satisfies :

(1) $f$ is an entire function in $Q^{\prime}$;

(2) $\int_{\mathbb{R}}|f(\xi)|^{2}\left(1+|\xi|^{2}\right)^{2} d \xi<\infty$

(3) $\forall \xi \in Q^{\prime} \quad|f(\xi)| \leq C(1+|\xi|)^{N} e^{L|\operatorname{Im} \xi|}$

for some positive constants $C, N$.

Since the roots of $\alpha-\beta e^{-i L \xi}$ are simple (unless $\alpha=\beta=0$ ), (1) holds provided that the roots of $\xi^{3}-\xi+p$ are simple and also roots of $\alpha-\beta e^{-i L \xi}$. Notice that if (1) holds true, then (2) and (3) are satisfied. It follows that $(\mathcal{A})$ is equivalent to the existence of complex numbers $p, \mu_{0}$ and of positive integers $k, l$ such that, if we set

$$
\mu_{1}:=\mu_{0}+k \frac{2 \pi}{L} \text { and } \mu_{2}:=\mu_{1}+l \frac{2 \pi}{L},
$$

we have

$$
\xi^{3}-\xi+p \equiv\left(\xi-\mu_{0}\right)\left(\xi-\mu_{1}\right)\left(\xi-\mu_{2}\right),
$$

that is

$$
\begin{aligned}
\mu_{0}+\mu_{1}+\mu_{2} & =0 \\
\mu_{0} \mu_{1}+\mu_{0} \mu_{2}+\mu_{1} \mu_{2} & =-1, \\
\mu_{0} \mu_{1} \mu_{2} & =-p .
\end{aligned}
$$

Easy calculations lead to

$$
\begin{aligned}
L & =2 \pi \sqrt{\frac{k^{2}+k l+l^{2}}{3}}, \\
\mu_{0} & =-\frac{1}{3}(2 k+l) \frac{2 \pi}{L}, \\
p & =-\mu_{0}\left(\mu_{0}+k \frac{2 \pi}{L}\right)\left(\mu_{0}+(k+l) \frac{2 \pi}{L}\right) .
\end{aligned}
$$

Hence $(\mathcal{A}) \Longleftrightarrow L \in \mathcal{N}$. This completes the proof of Lemmas 3.5, 3.4 and of Proposition 3.3. 
REMARK 3.6. (i) For $L \in \mathcal{N}$, if $p$ is given by (3.47) and $y_{0}$ (with $\operatorname{Re} y_{0} \neq 0$ ) is as in $(\mathcal{A})$ (with $\lambda=-i p$ ), then $y(t, x):=\operatorname{Re}\left(e^{-i p t} y_{0}(x)\right)$ is a nontrivial (smooth) solution of (3.5)-(3.8) such that $y_{x}(., 0) \equiv 0$. Thus the result in Proposition 3.3 holds true iff $L \notin \mathcal{N}$. On the other hand it may be shown (as in $[7$; remark 4.4, p.56]) that the existence of unobservable solutions of (3.5)-(3.8) leads to uncontrollability of equations (3.1)-(3.4).

(ii) In the particular situation where $k=l$, the system (3.45)-(3.47) reduces to $L=2 k \pi, \mu_{0}=-1, p=0$ (hence $\lambda=0$ ). This yields the (unobservable) steady solutions of (3.5)-(3.8): $y(t, x)=a(\cos x-1), a \in \mathbb{R}$.

(iii) If the term $y_{x}$ is removed from (3.5), it may be shown that Proposition 3.3 is true for every $L>0$.

The goal of the following proposition is to define -in a certain weak sensesolutions of the non-homogeneous problem (3.1)-(3.4).

Proposition 3.7. There exists a unique linear continuous map $\Psi: L^{2}(0, L)$ $\times L^{2}(0, T) \rightarrow B$ such that, for $y_{0} \in \mathcal{D}(A)$ and $h \in C^{2}([0, T])$ with $h(0)=0$, $\Psi\left(y_{0}, h\right)$ is the unique (classical) solution of (3.1)-(3.4).

Proof. Let $C_{0}^{2}([0, T]):=\left\{h \in C^{2}([0, T], I R) ; h(0)=0\right\}$.

First step: We assume here $y_{0} \in \mathcal{D}(A)$ and $h \in C_{0}^{2}([0, T])$. Let $\phi \in$ $C^{\infty}([0, L])$ be such that $\phi(0)=\phi(L)=0, \phi^{\prime}(L)=-1$. The change of function $z(t, x)=y(t, x)-\left(S(t) y_{0}\right)(x)+h(t) \phi(x)$ transforms (3.1)-(3.4) into

$$
\begin{aligned}
z_{t}+z_{x}+z_{x x x} & =f(t, x):=h^{\prime}(t) \phi(x)+h(t)\left(\phi^{\prime}(x)+\phi^{\prime \prime \prime}(x)\right) \\
z(t, 0)=z(t, L) & =0 \\
z_{x}(t, L) & =0 \\
z(0, x) & =0
\end{aligned}
$$

Since $f \in C^{1}\left([0, T], L^{2}(0, L)\right)$, there exists by Proposition 3.1 and a classical result concerning such non-homogeneous problems (see [15]) a unique solution

$$
z \in C([0, T], \mathcal{D}(A)) \cap C^{1}\left([0, T], L^{2}(0, L)\right)
$$

of (3.48)-(3.51). Hence for (smooth) data $y_{0} \in \mathcal{D}(A), h \in C_{0}^{2}([0,1])(3.1)$ (3.4) admits a unique (classical) solution

$$
y \in C\left([0, T], H^{3}(0, L)\right) \cap C^{1}\left([0, T], L^{2}(0, L)\right) .
$$

Second step: (A priori estimates by multiplier method.) We also assume $y_{0} \in \mathcal{D}(A), h \in C_{0}^{2}([0, T])$. Let $q \in C^{\infty}([0, T] \times[0, L])$. By $(3.52)$ we may integrate by parts in

$$
\int_{0}^{S} \int_{0}^{L} q y\left(y_{t}+y_{x}+y_{x x x}\right) d x d t=0 .
$$


( $S$ denotes some arbitrary number in $[0, T]$.) Using (3.1)-(3.4) (and Fubini's theorem) we get:

$$
\begin{aligned}
& -\int_{0}^{S} \int_{0}^{L}\left(q_{t}+q_{x}+q_{x x x}\right) \frac{y^{2}}{2} d x d t+\int_{0}^{L} \frac{\left(q y^{2}\right)(S, x)}{2} d x \\
& -\int_{0}^{L} \frac{\left(q y^{2}\right)(0, x)}{2} d x-\frac{1}{2} \int_{0}^{S} q(t, L) h(t)^{2} d t \\
& +\frac{3}{2} \int_{0}^{S} \int_{0}^{L} q_{x} y_{x}^{2} d x d t+\frac{1}{2} \int_{0}^{S}\left(q y_{x}^{2}\right)(t, 0) d t=0 .
\end{aligned}
$$

Choosing $q=1$ leads to

$$
\begin{gathered}
\int_{0}^{L} y(S, x)^{2} d x+\int_{0}^{S} y_{x}(t, 0)^{2} d t=\int_{0}^{L} y_{0}(x)^{2} d x+\int_{0}^{S} h(t)^{2} d t \\
\leq\left\|y_{0}\right\|_{L^{2}(0, L)}^{2}+\|h\|_{L^{2}(0, T)}^{2}
\end{gathered}
$$

Setting $\left\|\left(y_{0}, h\right)\right\|:=\left(\left\|y_{0}\right\|_{L^{2}(0, L)}^{2}+\|h\|_{L^{2}(0, T)}^{2}\right)^{\frac{1}{2}}$, we get

$$
\|y\|_{C\left([0, T], L^{2}(0, L)\right)} \leq\left\|\left(y_{0}, h\right)\right\|
$$

which yields

$$
\|y\|_{L^{2}((0, T) \times(0, L))} \leq \sqrt{T}\left\|\left(y_{0}, h\right)\right\|
$$

and

$$
\left\|y_{x}(., 0)\right\|_{L^{2}(0, T)} \leq\left\|\left(y_{0}, h\right)\right\| .
$$

Now take $q(t, x)=x$, and $S=T$. (3.54) gives

$$
\begin{gathered}
-\int_{0}^{T} \int_{0}^{L} \frac{y^{2}}{2} d x d t+\int_{0}^{L} x \frac{y(T, x)^{2}}{2} d x-\int_{0}^{L} x \frac{y_{0}(x)^{2}}{2} d x \\
+\frac{3}{2} \int_{0}^{T} \int_{0}^{L} y_{x}^{2} d x d t-\frac{1}{2} \int_{0}^{T} L h(t)^{2} d t=0 .
\end{gathered}
$$

Hence

$$
\int_{0}^{T} \int_{0}^{L} y_{x}^{2} d x d t \leq \frac{1}{3}\left(\int_{0}^{T} \int_{0}^{L} y^{2} d x d t+\int_{0}^{L} x y_{0}(x)^{2} d x+L \int_{0}^{T} h(t)^{2} d t\right)
$$

and then, using (3.57),

$$
\|y\|_{L^{2}\left(0, T, H^{1}(0, L)\right)} \leq\left(\frac{4 T+L}{3}\right)^{\frac{1}{2}}\left\|\left(y_{0}, h\right)\right\| .
$$

Using (3.56), (3.61), and the density of $\mathcal{D}(A)$ in $L^{2}(0, L)$ and of $C_{0}^{2}([0, T])$ in $L^{2}(0, T)$, we see that the linear map $\left(y_{0}, h\right) \in \mathcal{D}(A) \times C_{0}^{2}([0, T]) \mapsto y \in B$ may be extended in a unique manner to the whole space $L^{2}(0, L) \times L^{2}(0, T)$ to give a (linear) continuous map $\Psi: L^{2}(0, L) \times L^{2}(0, T) \rightarrow B$.

REMARK 3.8. i) For $y_{0} \in L^{2}(0, L)$ and $h \in L^{2}(0, T)$, the (weak) solution $\Psi\left(y_{0}, h\right)$ is solution of $(3.1)$ in $\mathcal{D}^{\prime}\left(0, T, H^{-2}(0, L)\right)$. Moreover $\Psi\left(y_{0}, h\right)(0,$. $\left(=y_{0}\right)$ and $\Psi\left(y_{0}, h\right)(T,$.$) are well-defined in L^{2}(0, L)$ since $\Psi\left(y_{0}, h\right) \in$ $C\left([0, T], L^{2}(0, L)\right)$.

ii) Obviously $\Psi\left(y_{0}, 0\right)=S(\cdot) y_{0}$, hence $\Psi\left(y_{0}, h\right)=S(\cdot) y_{0}+\Psi(0, h)$.

Esaim: CoCV, March 1997, Vol.2, PP. 33-55 
To apply H.U.M. we need some observability result concerning the following (backward well-posed) homogeneous problem:

$$
\begin{aligned}
u_{t}+u_{x}+u_{x x x} & =0 \\
u(t, 0)=u(t, L) & =0 \\
u_{x}(t, 0) & =0 \\
u(T, x) & =u_{T}(x) .
\end{aligned}
$$

(Notice that (3.64) (resp. (3.65)) differs from (3.7) (resp. (3.8)).) The change of variables $\tau=T-t, \xi=L-x$ transforms (3.62)-(3.65) into (3.5)(3.8) and vice-versa. Using Propositions $3.1,3.2$ and 3.3 we readily get the following result.

Proposition 3.9. Let $L, T>0$. For any $u_{T} \in L^{2}(0, L)$ the mild solution of (3.62)-(3.65) belongs to $B$, the function $u_{x}(., L)$ makes sense in $L^{2}(0, T)$. If moreover $L \notin \mathcal{N}$, there exists a constant $C=C(L, T)>0$ such that for any $u_{T} \in L^{2}(0, L)$ we have

$$
\left\|u_{x}(., L)\right\|_{L^{2}(0, T)} \leq\left\|u_{T}\right\|_{L^{2}(0, L)} \leq C\left\|u_{x}(., L)\right\|_{L^{2}(0, T)} .
$$

It remains to apply H.U.M.

Proof of Theorem 1.2. Thanks to Remark 3.8 ii), we may assume without loss of generality that $y_{0}=0$. (See the proof of Th. 1.1 for the details.) Let $\left(u_{T}, h\right) \in C_{c}^{\infty}(0, L) \times C_{c}^{\infty}(0, T)$, let $u$ (resp. $\left.y\right)$ be the (classical) solution of (3.62)-(3.65) (resp. (3.1)-(3.4)). Integrating by parts in

$$
\int_{0}^{T} \int_{0}^{L} u\left(y_{t}+y_{x}+y_{x x x}\right) d x d t=0,
$$

we readily get

$$
\int_{0}^{L} u_{T}(x) y(T, x) d x=\int_{0}^{T} u_{x}(t, L) h(t) d t .
$$

By an argument of density we see that (3.68) holds true for $u_{T} \in L^{2}(0, L)$ and $h \in L^{2}(0, T)$. Let $\Lambda$ denote the (linear) continuous map

$$
u_{T} \in L^{2}(0, L) \mapsto y(T, .) \in L^{2}(0, L),
$$

$y$ standing for the solution of (3.1)-(3.4) associated with the data $h:=$ $u_{x}(., L) \in L^{2}(0, T)$. It follows from (3.68) and Prop. 3.9 that

$$
\left(\Lambda\left(u_{T}\right), u_{T}\right)_{L^{2}(0, L)}=\left\|u_{x}(., L)\right\|_{L^{2}(0, T)}^{2} \geq C^{-2}\left\|u_{T}\right\|_{L^{2}(0, L)}^{2} .
$$

Hence $\Lambda$ is invertible by Lax-Milgram theorem. The proof of Theorem 1.2 is complete.

REMARK 3.10. When $y_{0}=0$, H.U.M. yields a (linear) continuous selection of the control, namely the map

$$
\Gamma: y_{T} \in L^{2}(0, L) \mapsto u_{x}(., L) \in L^{2}(0, T),
$$

where $u$ denotes the solution of (3.62)-(3.65) associated with $u_{T}:=\Lambda^{-1}\left(y_{T}\right)$. 


\section{EXACT BOUNDARY CONTROLLABILITY OF NONLINEAR KDV EQUATION ON A BOUNDED DOMAIN}

In this section we prove that the following boundary-control system

$$
\begin{aligned}
y_{t}+y_{x}+y y_{x}+y_{x x x} & =0 \\
y(t, 0)=y(t, L) & =0 \\
y_{x}(t, L) & =h(t) \quad\left(h \in L^{2}(0, T)\right) \\
y(0, x) & =y_{0}(x)
\end{aligned}
$$

is exactly controllable in a neighbourhood of the nul state. More precisely we show that for any $L>0$ and $T>0$ there exists a radius $r_{0}>0$ such that for every $y_{0}, y_{T} \in L^{2}(0, L)$ with $\left\|y_{0}\right\|_{L^{2}(0, L)}<r_{0},\left\|y_{T}\right\|_{L^{2}(0, L)}<r_{0}$ we may find $y \in B=C\left([0, T], L^{2}(0, L)\right) \cap L^{2}\left(0, T, H^{1}(0, L)\right)$ such that 1$) y_{t}=$ $-\left(y_{x}+y y_{x}+y_{x x x}\right)$ in $\mathcal{D}^{\prime}\left(0, T, H^{-2}(0, L)\right)$ and 2) $y(0,)=.y_{0}, y(T,)=.y_{T}$. (Notice that for $y \in B, y_{x} \in L^{2}\left(0, T, L^{2}(0, L)\right), y_{x x x} \in L^{2}\left(0, T, H^{-2}(0, L)\right)$ and $y y_{x} \in L^{1}\left(0, T, L^{2}(0, L)\right)$ - see below Prop. 4.1 - hence $y_{x}+y y_{x}+y_{x x x} \in$ $L^{1}\left(0, T, H^{-2}(0, L)\right)$.) If moreover $L \notin \mathcal{N}$, then $(4.2)$ is true in $L^{2}(0, T)$ and (4.3) holds in a weak sense (that we will not specify) for some control function $h \in L^{2}(0, T)$.

To solve (4.1)-(4.4), we write $y=S(t) y_{0}+y_{1}+y_{2}$ where $(S(t))_{t>0}$ denotes the semi-group associated with the operator $A$ of section $3, y_{1}$ and $y_{2}$ are (respective) solutions of two nonhomogeneous problems:

$$
\begin{aligned}
y_{1 t}+y_{1 x}+y_{1 x x x} & =0 \\
y_{1}(t, 0)=y_{1}(t, L) & =0 \\
y_{1 x}(t, L) & =h(t) \\
y_{1}(0, x) & =0
\end{aligned}
$$

and

$$
\begin{aligned}
y_{2 t}+y_{2 x}+y_{2 x x x} & =f \\
y_{2}(t, 0)=y_{2}(t, L) & =0 \\
y_{2 x}(t, L) & =0 \\
y_{2}(0, x) & =0 .
\end{aligned}
$$

(In (4.9) we have set $f=-y y_{x}$. ) Let $\psi_{1}: h \in L^{2}(0, T) \mapsto y_{1} \in B$ be the map which associates with $h$ the weak solution of (4.5)-(4.8). By Proposition $3.7, \psi_{1}$ is a (linear) continuous map. The following proposition deals with equations (4.9)-(4.12):

Proposition 4.1. 1) If $y \in L^{2}\left(0, T, H^{1}(0, L)\right), y y_{x} \in L^{1}\left(0, T, L^{2}(0, L)\right)$ and the map $y \mapsto y y_{x}$ is continuous.

2) For $f \in L^{1}\left(0, T, L^{2}(0, L)\right)$ the mild solution $y_{2}$ of (4.9)-(4.12) belongs to $B$. Moreover the linear map $\psi_{2}: f \mapsto y_{2}$ is continuous.

REMARK 4.2. Recall that for $f \in L^{1}\left(0, T, L^{2}(0, L)\right)$ the mild solution $y_{2}$ of (4.9)-(4.12) is given by

$$
y_{2}(t, .):=\int_{0}^{t} S(t-s) f(s, .) d s .
$$


Proof. 1) Let $y, z \in L^{2}\left(0, T, H^{1}(0, L)\right)$. Let $C_{1}$ denote the norm of the Sobolev embedding $H^{1}(0, L) \hookrightarrow L^{\infty}(0, L)$. Applying triangular inequality and Hölder's inequality we get

$$
\begin{aligned}
\| y y_{x} & -z z_{x} \|_{L^{1}\left(0, T, L^{2}(0, L)\right)} \\
\leq & \int_{0}^{T}\left\|(y-z) y_{x}(t, .)\right\|_{L^{2}(0, L)} d t+\int_{0}^{T}\left\|z\left(y_{x}-z_{x}\right)(t, .)\right\|_{L^{2}(0, L)} d t \\
\leq & \int_{0}^{T}\|(y-z)(t, .)\|_{L^{\infty}(0, L)}\left\|y_{x}(t, .)\right\|_{L^{2}(0, L)} d t \\
& +\int_{0}^{T}\|z(t, .)\|_{L^{\infty}(0, L)}\left\|\left(y_{x}-z_{x}\right)(t, .)\right\|_{L^{2}(0, L)} d t \\
\leq & C_{1}\left(\int_{0}^{T}\|(y-z)(t, .)\|_{H^{1}(0, L)}\|y(t, .)\|_{H^{1}(0, L)} d t\right. \\
& \left.+\int_{0}^{T}\|z(t, .)\|_{H^{1}(0, L)}\|(y-z)(t, .)\|_{H^{1}(0, L)} d t\right) \\
\leq & C_{1}\left(\|y\|_{L^{2}\left(0, T, H^{1}(0, L)\right)}\right. \\
& \left.+\|z\|_{L^{2}\left(0, T, H^{1}(0, L)\right)}\right)\|y-z\|_{L^{2}\left(0, T, H^{1}(0, L)\right)} .
\end{aligned}
$$

(4.14) with $z=0$ yields $y y_{x} \in L^{1}\left(0, T, L^{2}(0, L)\right)$, and (4.14) with $z$ tending to $y$ gives the continuity of the map $y \mapsto y y_{x}$.

2) Since

$$
\left\|1_{[0, t]}(s) S(t-s) f(s, .)\right\|_{L^{2}(0, L)} \leq\|f(s, .)\|_{L^{2}(0, L)} \in L^{1}(0, T)
$$

it follows from Lebesgue's theorem that the mild solution $y_{2}(t,)=$. $\int_{0}^{t} S(t-s) f(s,) d$.$s belongs to C\left([0, T], L^{2}(0, L)\right)$. Moreover for every $t \in$ $[0, T]$

$$
\left\|y_{2}(t, .)\right\|_{L^{2}(0, L)} \leq \int_{0}^{t}\|f(s, .)\|_{L^{2}(0, L)} d s \leq\|f\|_{L^{1}\left(0, T, L^{2}(0, L)\right)}
$$

so the linear map $f \in L^{1}\left(0, T, L^{2}(0, L)\right) \mapsto y_{2} \in C\left([0, T], L^{2}(0, L)\right)$ is continuous. To show this map is (well-defined and) continuous from $L^{1}\left(0, T, L^{2}(0, L)\right)$ into $L^{2}\left(0, T, H^{1}(0, L)\right)$, it is clearly sufficient to prove:

$$
\begin{aligned}
& \exists C_{2}>0, \forall f \in C^{1}\left([0, T], L^{2}(0, L)\right) \\
& \left\|y_{2 x}\right\|_{L^{2}((0, T) \times(0, L))} \leq C_{2}\|f\|_{L^{1}\left(0, T, L^{2}(0, L)\right)}
\end{aligned}
$$

Integrating by parts in

$$
\int_{0}^{T} \int_{0}^{L} x y_{2}\left(y_{2 t}+y_{2 x}+y_{2 x x x}\right) d x d t=\int_{0}^{T} \int_{0}^{L} x y_{2} f d x d t
$$


we get

$$
\begin{aligned}
\int_{0}^{L} x \frac{y_{2}(T, x)^{2}}{2} d x & -\int_{0}^{T} \int_{0}^{L} \frac{y_{2}(t, x)^{2}}{2} d x d t \\
& +\frac{3}{2} \int_{0}^{T} \int_{0}^{L} y_{2 x}(t, x)^{2} d x d t \\
& =\int_{0}^{T} \int_{0}^{L} x\left(f y_{2}\right)(t, x) d x d t
\end{aligned}
$$

Hence, using (4.16)

$$
\begin{aligned}
& \int_{0}^{T} \int_{0}^{L} y_{2 x}(t, x)^{2} d x d t \\
\leq & \frac{1}{3} \int_{0}^{T} \int_{0}^{L} y_{2}(t, x)^{2} d x d t+\frac{2}{3} L \int_{0}^{T}\|f(t, .)\|_{L^{2}(0, L)} \cdot\left\|y_{2}(t, .)\right\|_{L^{2}(0, L)} d t \\
\leq & \frac{1}{3} T\|f\|_{L^{1}\left(0, T, L^{2}(0, L)\right)}^{2}+\frac{2}{3} L\|f\|_{L^{1}\left(0, T, L^{2}(0, L)\right)} \cdot\left\|y_{2}\right\|_{C\left([0, T], L^{2}(0, L)\right)} \\
\leq & \frac{1}{3}(T+2 L)\|f\|_{L^{1}\left(0, T, L^{2}(0, L)\right)}^{2} .
\end{aligned}
$$

The proof of Proposition 4.1 is complete.

We may now prove the main result of this paper.

Proof of Theorem 1.3. We first assume that $L \notin \mathcal{N}$. We show that for $T>0$ there exists $r_{0}>0$ (small enough) such that if $\left\|y_{0}\right\|_{L^{2}(0, L)},\left\|y_{T}\right\|_{L^{2}(0, L)}<r_{0}$, the state $y_{T}$ may be reached from $y_{0}$ for nonlinear KdV equation. Let $y_{0}, y_{T}$ be states in $L^{2}(0, L)$ such that $\left\|y_{0}\right\|_{L^{2}(0, L)},\left\|y_{T}\right\|_{L^{2}(0, L)} \leq r, r>0$ to be chosen later. Let $F$ denote the nonlinear map

$$
\begin{gathered}
y \in L^{2}\left(0, T, H^{1}(0, L)\right) \mapsto F(y) \\
:=S(.) y_{0}+\psi_{1} \circ \Gamma\left(y_{T}-S(T) y_{0}+\psi_{2}\left(y y_{x}\right)(T, .)\right)+\psi_{2}\left(-y y_{x}\right) \in B(4.21)
\end{gathered}
$$

( $\Gamma$ is defined in Remark 3.10, $\psi_{1}$ and $\psi_{2}$ are defined at the beginning of this section.) $\quad F$ is well-defined and continuous by Propositions 3.2, 3.7, 4.1 and Remark 3.10. Clearly each fixed point of $F$ verifies (4.1) in $\mathcal{D}^{\prime}\left(0, T, H^{-2}(0, L)\right)$ -,$(4.4)$ and $y(T,)=.y_{T}$. For proving existence of fixed-point for $F$ we apply Banach contraction fixed-point theorem to the restriction of $F$ to some closed ball $\bar{B}(0, R)$ in $L^{2}\left(0, T, H^{1}(0, L)\right)$ ( $R$ will be chosen later.) We need:

$$
F(\bar{B}(0, R)) \subset \bar{B}(0, R)
$$

and

$$
\left.\exists C_{3} \in\right] 0,1\left[, \forall y, z \in \bar{B}(0, R) \quad\|F(y)-F(z)\| \leq C_{3}\|y-z\|,\right.
$$

where $\|$.$\| from now on stands for the norm L^{2}\left(0, T, H^{1}(0, L)\right)$. Let $K_{1}$ (resp. $K_{2}, K_{2}^{\prime}$ ) denote the norm of $\psi_{1}$ (resp. $\psi_{2}, \psi_{2}$ ) as a map from $L^{2}(0, T)$ (resp. $\left.L^{1}\left(0, T, L^{2}(0, L)\right)\right)$ into $L^{2}\left(0, T, H^{1}(0, L)\right)\left(\operatorname{resp} . L^{2}\left(0, T, H^{1}(0, L)\right)\right.$, $\left.C\left([0, T], L^{2}(0, L)\right)\right)$, and let $K$ denote the norm of $\Gamma$ as a map from $L^{2}(0, L)$ into $L^{2}(0, T)$. Set $K_{3}=\sqrt{\frac{4 T+L}{3}}$. Let $y, z \in L^{2}\left(0, T, H^{1}(0, L)\right)$. Assume

$$
\|y\| \leq R,\|z\| \leq R .
$$


Then by (3.19) and (4.14)

$$
\begin{aligned}
\|F(y)\| & \leq \sqrt{\frac{4 T+L}{3}}\left\|y_{0}\right\|_{L^{2}(0, L)}+K_{1} K\left(\left\|y_{T}\right\|_{L^{2}(0, L)}\right. \\
& \left.+\left\|y_{0}\right\|_{L^{2}(0, L)}+K_{2}^{\prime} C_{1}\|y\|^{2}\right)+K_{2} C_{1}\|y\|^{2} \\
& \leq C_{1}\left(K_{2}+K K_{1} K_{2}^{\prime}\right) R^{2}+\left(2 K K_{1}+K_{3}\right) r .
\end{aligned}
$$

(4.22) leads to the first condition on $R, r$ :

$$
C_{1}\left(K_{2}+K K_{1} K_{2}^{\prime}\right) R^{2}+\left(2 K K_{1}+K_{3}\right) r \leq R
$$

Now write

$$
F(y)-F(z)=\psi_{2}\left(z z_{x}-y y_{x}\right)+\psi_{1} \circ \Gamma\left(\psi_{2}\left(y y_{x}-z z_{x}\right)(T, .)\right) .
$$

We get, by (4.14),

$$
\|F(y)-F(z)\| \leq 2 C_{1}\left(K_{2}+K K_{1} K_{2}^{\prime}\right) R\|y-z\| .
$$

(4.23) will hold provided that

$$
2 C_{1}\left(K_{2}+K K_{1} K_{2}^{\prime}\right) R<1 .
$$

Let $R$ be some positive number verifying (4.29). Then (4.26) holds true if we take

$$
r:=\frac{R}{2\left(2 K K_{1}+K_{3}\right)} .
$$

Setting

$$
r_{0}:=\left(4 C_{1}\left(2 K K_{1}+K_{3}\right)\left(K_{2}+K K_{1} K_{2}^{\prime}\right)\right)^{-1}
$$

we see that $r \rightarrow r_{0}$ as $R \rightarrow\left(2 C_{1}\left(K_{2}+K K_{1} K_{2}^{\prime}\right)\right)^{-1}$. It follows that if $\left\|y_{0}\right\|_{L^{2}(0, L)}<r_{0}$ every $y_{T}$ with $\left\|y_{T}\right\|_{L^{2}(0, L)}<r_{0}$ may be reached by a solution of nonlinear KdV equation coming from $y_{0}$. The proof of Theorem 1.3 is achieved when $L \notin \mathcal{N}$. If now $L \in \mathcal{N}$, it is sufficient to consider some $\bar{L}>L$ such that $\bar{L} \notin \mathcal{N}$ and to apply the theorem to the functions $\bar{y}_{0}, \bar{y}_{T} \in$ $L^{2}(0, \bar{L})$, where $\bar{y}_{0}, \bar{y}_{T}$ denote the prolongations (by 0 ) of (given) states $y_{0}$, $y_{T} \in L^{2}(0, L)$, and then to restrict the solution $\bar{y}$ to the domain $(0, T) \times$ $(0, L)$. The proof of Theorem 1.3 is complete.

REMARK 4.3. As it has been pointed out to the author by E. Zuazua, the linear KdV equation is exactly boundary controllable when $L \in \mathcal{N}$ if $y(., L)$ is also assumed to be controlled. More precisely let $k \in N^{*}, L>0$ and $T>0$. Then for any $y_{0}, y_{T} \in L^{2}(0, L)$ there exist $h_{1} \in H_{0}^{k}(0, T)$ and $h_{2} \in L^{2}(0, T)$ such that the generalized solution of the initial-boundary-value problem

$$
\begin{aligned}
y_{t}+y_{x}+y_{x x x} & =0 \\
y(t, 0) & =0 \\
y(t, L) & =h_{1}(t) \\
y_{x}(t, L) & =h_{2}(t) \\
y(0, x) & =y_{0}(x)
\end{aligned}
$$

satisfies $y(T,)=.y_{T}$. Indeed it may be shown that (for any $L>0$ ) there exist positive constants $C_{1}, C_{2}$ such that for every $u_{T} \in L^{2}(0, L)$, if $u$ denotes 
the solution of (3.62)-(3.65), then:

$$
\begin{array}{r}
C_{1}\left(\left\|u_{x}(., L)\right\|_{L^{2}(0, T)}+\left\|u_{x x}(., L)\right\|_{H^{-k}(0, T)}\right) \\
\leq\left\|u_{T}\right\|_{L^{2}(0, L)} \leq \quad C_{2}\left(\left\|u_{x}(., L)\right\|_{L^{2}(0, T)}+\left\|u_{x x}(., L)\right\|_{H^{-k}(0, T)}\right) .
\end{array}
$$

(Lemma 3.4 holds true this time for every $L>0$ since $y_{0} \in H^{3}(0, L)$, $\lambda y_{0}=-y_{0}{ }^{\prime}-y_{0}{ }^{\prime \prime \prime}$ and $y_{0}(0)=y_{0}{ }^{\prime}(0)=y_{0}{ }^{\prime \prime}(0)=0$ imply $y_{0}=0$.) Proceeding as in section 4, we get the following (alternative) statement of Theorem 1.3: Let $k \in N^{*}, L>0$ and $T>0$. Then if $y_{0}$ and $y_{T}$ are given states sufficiently close to 0 in $L^{2}(0, L)$, there exist $h_{1} \in H_{0}^{k}(0, T)$ and $h_{2} \in L^{2}(0, T)$ such that the generalized solution $y \in C\left([0, T], L^{2}(0, L)\right) \cap L^{2}\left(0, T, H^{1}(0, L)\right)$ of

$$
\begin{aligned}
y_{t}+y_{x}+y y_{x}+y_{x x x} & =0 \\
y(t, 0) & =0 \\
y(t, L) & =h_{1}(t) \\
y_{x}(t, L) & =h_{2}(t) \\
y(0, x) & =y_{0}(x)
\end{aligned}
$$

satisfies $y(T,)=.y_{T}$.

The author thanks the Dipartimento di Matematica of Politecnico Di Torino for its hospitality. The author also likes to thank J.M. Coron for bringing his attention to the boundary controllability of KdV and E. Zuazua for pointing to him techniques permitting to drop useless assumptions.

\section{REFERENCES}

[1] C. Bardos, G. Lebeau and J. Rauch: Sharp sufficient conditions for the observation, control and stabilization of waves from the boundary, SIAM J. Control Optim., 30 , $1992,1024-1065$.

[2] J. Bona and R. Winther: The Korteweg-de Vries equation, posed in a quarter-plane, SIAM J. Math Anal., 14, 1983, 1056-1106.

[3] J.M. Coron: Contrôlabilité exacte frontière de l'équation d'Euler des fluides parfaits incompressibles bidimensionnels, C. R. Acad. Sci. Paris, t. 317, Série I, 1993, 271276.

[4] A.V. Fursikov and O.Y. Imanuvilov: On controllability of certain systems simulating a fluid flow, in Flow Control, IMA, Math. Appl., vol. 68, Gunzberger ed., SpringerVerlag, New York, 1995, 148-184.

[5] L.F. Ho: Observabilité frontière de l'équation des ondes, C. R. Acad. Sci. Paris, Série 1 Math., 302, 1986, 443-446.

[6] A.E. Ingham: Some trigonometrical inequalities with application to the theory of series, Math. A., 41, 1936, 367-379.

[7] V. Komornik: Exact controllability and stabilization, the multiplier method, R.A.M. 36, John Wiley-Masson, 1994.

[8] V. Komornik, D.L. Russel and B.-Y. Zhang: Control and stabilization of the Korteweg-de Vries equation on a periodic domain, submitted to J. Differential Equations.

[9] D.J. Korteweg and G. de Vries: On the change of form of long waves advancing in a rectangular canal, and on a new type of long stationary waves, Philos. Mag., 5, 39, 1895, 422-423.

[10] G. Lebeau: Contrôle de l'équation de Schrödinger, J. Math. Pures Appl., 71, 1992, $267-291$.

[11] J.L. Lions: Contrôlabilité exacte de systèmes distribués, C.R. Acad. Sci. Paris, 302, $1986,471-475$. 
[12] J.L. Lions: Contrôlabilité exacte, Perturbations et Stabilisation de Systèmes Distribués; Tome 1, Contrôlabilité exacte, Collection de recherche en mathématiques appliquées, 8, Masson, Paris, 1988.

[13] J.L. Lions: Exact controllability, stabilizability, and perturbations for distributed systems, Siam Rev., 30, 1988, 1-68.

[14] E. Machtyngier: Exact controllability for the Schrödinger equation, SIAM J. Control Optim., 32, 1994, 24-34.

[15] A. Pazy: Semigroups of linear operators and applications to partial differential equations, Springer-Verlag, New York, 1983.

[16] D.L. Russel and B-Y. Zhang: Controllability and stabilizability of the third-order linear dispersion equation on a periodic domain, SIAM J. Control Optim., 31, 1993, 659-673.

[17] J.C. Saut and R. Temam: Remarks on the Korteweg-De Vries equation, Israel. Math., 24, 1976, 78-87.

[18] J. Simon: Compact sets in the space $L^{p}(0, T, B)$, Annali di Matematica pura ed applicata (IV), vol. CXLVI, 1987, 65-96.

[19] K. Yosida: Functional Analysis, Springer-Verlag, Berlin Heidelberg New York, 1978.

[20] B.-Y. Zhang: Some results for nonlinear dispersive wave equations with applications to control, Ph. D. thesis, University of Wisconsin, Madison, June 1990. 